\title{
Problems of social and psychological adaptation of students in conditions of social deprivation
}

\author{
Elena Dvoinikova ${ }^{1 *}$, Ekaterina Bakshutova ${ }^{1}$, Natalya Beylina ${ }^{1}$, Olga Telnova $^{2}$, and Julia \\ Vasilieva $^{1}$ \\ ${ }^{1}$ Samara State Technical University, 443100, Samara, Russia \\ ${ }^{2}$ Southern Federal University, 344000, Rostov-on-Don, Russia
}

\begin{abstract}
The article is devoted to changes in the level of empathy and some structural components of social and psychological adaptation caused by social deprivation in a pandemic, which is the purpose of the work. The paper presents the results of an empirical study conducted in the first semester of the 2020-2021 academic year, when Samara State Technical University students were on distance learning. The sample consisted of 248 first-year students. The basis of the observation is a longitudinal research, during which the communicative activity of students in interaction with the teacher and with each other was recorded. In addition, students solved situational problems containing a description of the conflict, for a way out of which they had to offer solutions: none, one or several. The study confirmed the hypothesis put forward about possible changes in the level of empathy, the ability to solve situational problems and social and psychological adaptation in general among students studying remotely. Isolation in a pandemic is an objective measure for which humanity was not ready.
\end{abstract}

\section{Introduction}

We tried to ask a passer-by on the street (a neighbor, friend, relative, work colleague) with the question of what deprivation is. Today people know many words that describe human psychology. They know (have heard) about bipolar disorder, abuse, stress, disadaptation, and many others. But you don't often hear about deprivation. And this is all the more surprising that most of us, especially those who are engaged in intellectual work, are in isolation, and therefore in conditions of social deprivation. This circumstance requires deep reflection (and self-reflection).

The researchers focus on social and psychological adaptation (SPA), empathy and deprivation - more precisely, on what happens to the first named phenomena of a person's life in conditions of social isolation and deprivation. The problem of SPA has become more urgent in conditions of social isolation, dictated by the threat of infection with the virus COVID-19. A person is social, therefore, restrictions that exclude his natural communications affect not only the emotional sphere of the psyche, but also its functioning, and since for full mental development a person needs to receive various incentives:

\footnotetext{
* Corresponding author: dey10@rambler.ru
} 
emotional, sensory and others, their deprivation leads to a state deprivation due to the inability to satisfy mental needs completely. Self-isolation as a voluntary refusal from extensive natural communications in order to maintain health safety during a pandemic belongs to the category of voluntary-forced social deprivation. Since self-isolation was introduced in an emergency mode, the usual way of life of people has changed dramatically. In such conditions, the level of dissatisfaction with the environment increases, many people lose control over themselves, as well as the meaning and purpose of life, as a result of such conditions, apathy and passivity arise.

The purpose of this study is to study social deprivation as a factor in changing the SPA of students studying online in a pandemic. SPA has structural components, the indicators of which are markers of the success of adaptation. As a hypothesis, we put forward the assumption about changes in students' levels of indicators of the structural components of SPA and empathy.

First of all, in order to achieve the goal of the study, we carried out a theoretical analysis of scientific ideas on the problems of adaptation, empathy and deprivation.

In the works of the 30 s of the XX century, when the state of deprivation had just begun to be studied, it was about "maternal" deprivation. Scholars, studying this phenomenon, analyzed the state of children in various boarding schools, since such children had a lag in development, which was associated with a lack of emotional communication with close adults. Modern researchers began to consider the concept of "social deprivation" more broadly. But in general, the psychological literature does not yet have a unified interpretation of the term "deprivation", but most likely conventionality in its understanding has been achieved. Translated from English, this term means "deprivation, loss". In Russian, there are the following synonyms for this term: "mental starvation", "mental insufficiency", "mental deprivation". The most comprehensive interpretation of this concept was proposed by the researchers J. Langmeyer and Z. Matejček. "Mental deprivation is a mental state that has arisen as a result of such life situations where the subject is not given the opportunity to satisfy some of his basic (life) mental needs in a sufficient measure and for a sufficiently long time" [1, p. 246]. We will base on the classification proposed by them in their work. The authors identified four types of deprivation: stimulus (sensory), cognitive, emotional, and social.

In some scientific sources, sensory deprivation is described using the term "depleted environment". A "depleted environment" is understood as an environment in which a person does not receive enough various incentives - auditory, visual, tactile and others. Both a child and an adult can face this type of deprivation.

Cognitive or informational deprivation does not allow creating adequate models of the world. With this type of deprivation, a person does not possess important and necessary information for an accurate understanding of the connections that arise between objects and phenomena, therefore, according to I.P. Pavlova, he begins to create "imaginary connections", he has false judgments and beliefs that distort the real picture of the world [2].

Emotional deprivation can be experienced by people of all ages throughout their lives. In children, this type of deprivation is often inseparably linked with "maternal deprivation", since the mother plays a very important role in the emotional development of the child. It should be noted that even a slight deficit of emotional connection with the mother can lead to serious psychological problems in the future, as well as to various deviations of the child's psyche.

It is also necessary to note the motofacient deprivation that a person encounters in a situation of restriction in movement or transportation (injury, illness, etc.). This type of deprivation is not mental, but despite this it is quite closely related to it, since restrictions on movement have a strong enough effect on the emotional (mental) condition of a person, as well as on the psychological well-being of the individual in general. 
One of the most common types of deprivation is social, which can be forced, compulsory, voluntary, voluntary-forced. In any case, a person cannot fully satisfy the need of interaction with other people (limited circle of contacts). All these types of deprivation manifested themselves in the situation of the 2020-2021 pandemic [3].

The next aspect of this study is adaptation. Interest in the study of adaptation issues has not lost its relevance since the middle of the 19th century, when the development of natural and social sciences motivated people to acquire new competencies for social survival. However, the semantic context of adaptation, being unified for different scientific schools and meaning the adaptation of the object to the requirements of the environment [4], turned out to be insufficient for psychological science, since the phenomenon of the human personality is so many-sided that unified concepts cannot reflect all the meaningful aspects of a person's life. In the framework of further studies of human psychology, it was concluded that the adaptation of the personality is characterized by an active-adaptive nature. So, in the study of adaptation, new approaches to its consideration appeared.

At the beginning of the 20th century, representatives of psychomarkism A. Pauli and R. France represented adaptation through the inclusion of the need-motivational sphere of a person's personality to achieve a condition of adaptiveness [5]. In psychological studies of adaptation, the main conceptual approaches to the consideration of the phenomenon of adaptation, in particular psychophysiological, in which it is presented in the form of changes in the environment and changes in the body through actions corresponding to a given situation (R. Hankey) [quote by: 6]. In the works of Z. Freud and A. Freud, adaptation is understood as a model of the structure of the mental sphere of a person, the structural components of which are "Id", "Ego", "Super-Ego". In the case of a conflict between "Id" and "Super-Ego", the role of "Ego" is to fulfill the requests of "Id" without coming into conflict with the "Super-Ego", thus avoiding the occurrence of intrapersonal conflict [7, 8]. Considering the structure of consciousness, K.G. Jung defined adaptation as the main function of consciousness broader than the concept of "cognition", and which can be realized not only in cognitive activity [9]. In the concept of G. Hartman, psychoanalysis makes it possible to identify the processes that are the result of the impact of the environment, causing the condition of adaptability of the individual [10].

Behaviorists introduced the term "social adaptation", which implies not only the result, but also the process by which an individual or a group comes to a condition of social equilibrium or lack of experience of conflict with the environment [11]. A.A. Nalchajyan notes that such a concept takes into account conflicts with the external environment, while intrapersonal conflicts are ignored [11]. Later, the factor of emotional condition was included in the content of understanding the mechanism of adaptation [11]. In this direction, G. Selye developed a theory of the general adaptative syndrome, which was aimed at overcoming the negative effect of extraordinary incentives, for some time increasing "nonspecific resistance" [12]. The stressor in this theory was the cause of the general adaptative syndrome, which manifested itself as a response to stress.

The interactionist's concept of adaptation, set out by L. Phillips [11], considers adaptation as a result of the action of intrapsychic and environmental factors. Effective adaptation is "that kind of adaptation, upon achievement of which a person meets the minimum requirements and expectations of society" [11, p. 10]. In the theory of T. Shibutani, each person is endowed with a complex of techniques for solving difficulties and are considered as forms of adaptation [6]. Within the framework of the social and psychological approach, a person is an object and subject of social relations, under the influence of which he develops. A.A. Rean presents adaptation as the "involvement" of the individual in the social environment, as a system of values, norms and relations [13]. 
Thus, in modern psychology, adaptation is considered as a many-sided, active process of mastering the environment by an individual in order to assimilate its conditions through training and transformation of these conditions.

The phenomenon of empathy among those studied by us seems to be the most sympathetic. Empathy (Greek غ̉v - "in" + Greek Пá $\theta$ o - "passion", "suffering", "feeling" conscious empathy with the current emotional condition of another person without losing the feeling of the external origin of this emotional experience [14]. The ability to empathy is the norm, and as empaths, we empathize with any emotions and emotional conditions of people, and not just, for example, their sadness. Empathy itself is more a process than a condition, and it is more often described in fiction than in scientific works. Empathy doesn't mean only emotions. Like other mental phenomena, it has a complex structure - the cognitive aspect (we understand the emotions of other people, while realizing that these are the emotions of another person), emotional (we empathize, imbued with the emotion of another person), behavioral, conative (we do something for another person or for ourselves in connection with certain emotional experiences.) Nowadays there are quite a lot of works related to the manifestations of empathy in medical situations, e.g.: "The relationship between parental control and subclinical depressive symptoms in college freshmen: the role of empathy and gender" [15], "Neural characteristics of the empathic responses of parents imagining the suffering of their adolescent child" [16], etc.

J. Zaki writes: "And in the process of evolution, the brain has learned to better understand the thoughts and feelings of others. As a result, we have acquired numerous empathic abilities. We can open the door to the minds of not only friends and neighbors, but also enemies, strangers and even characters from movies and books that do not exist in reality. This is how we became the kindest species on the planet. Chimpanzees, for example, can act in an organized manner and console each other in sorrow, but their kindness is not unlimited. They rarely share food and, although friendly to members of their pack, they will not show mercy to outsiders. In contrast, people are world champions in cooperation and more than other species tend to help each other. This is our secret weapon" [17, p. 6]. But she also notes a decrease in the level of empathy among modern people, especially due to Internet communications: while remaining anonymous, we do not see each other's true faces, we do not hear voices, and we can hurt.

Since our sociality has become largely distant, we need to understand what is happening with our empathy.

\section{Materials and methods}

To determine the characteristics of SPA and empathy in conditions of social deprivation, we conducted a study of the SPA of students and their empathic abilities. The experiment involved 248 first-year students of the Samara State Technical University, studying psychological disciplines. The observation was carried out for four months in the fall semester of the 2020-2021 academic year.

In this study, one of the problems was to record the results of completing educational tasks and the activity of communication with the teacher and between students in the process of online learning. The activity of communications was recorded during longitudinal inside observation of the activity of students in dialogues with the teacher and among themselves, in the process of solving situational tasks: at the beginning of training there was an active discussion, a search for answers to the teacher's questions; at the end of the semester - waiting for answers from other students, or answers "I don't know", or "it cannot be solved".

Solving situational tasks - finding the best strategy for regulating the processes of a stressful situation in the task (tasks were compiled by E.Yu. Dvoinikova). The complex of situational tasks had three levels of complexity, each of which proposed a problem situation 
and a set of questions about its diagnosis, forecast and resolution options; the indicator of the success of such tasks was the variability of such solutions. The success of solving situational problems was determined by the proposed strategies and the number of solution options. The condition of the task provides for finding such solutions that would lead to the satisfaction of both sides of the participants in the situation: 1-2 solutions - low variability; 3-4 - medium and 5 or more - high.

Example of a Level 1 Problem:

During the period of fixing the attendance of classes by students to derive the rating of the groups, one of the students missed classes without a good reason and did not complete educational tasks. Due to his violation of discipline, the group lost rating status, and the students did not receive encouragement. In a conversation with groupmates about what happened, the student accused the university direction of the bias of the decision. In his opinion, he should have suffered alone.

Example of a Level 2 Problem:

Whenever an employee, in a conversation with a boss, tries to highlight a personal problem that is causing him to be unable to work overtime, the boss naturally changes the topic of the conversation. The employee knows about his rights and tries to defend them in a conversation with the manager, but he pretends that he does not see any problems in the situation told by the employee and continues to load him with work.

Example of a Level 3 Problem:

After a long period of crisis, a specialist finds a highly paid job in his specialty, which requires an irregular presence at the workplace and frequent business trips. The wife opposes such work and each time meeting him from work, demonstrates her dissatisfaction, announcing her jealousy and the ability to end family relationships.

Diagnostics of the conditions of the subjects of solving situational tasks showed their empathic abilities; the prediction of the development of events and the variability of permissions demonstrated social competence, conditioned by the SPA and empathy of the students.

The testing procedure using the methodology for diagnosing personality SPA by K. Rogers and R. Diamond [18] made it possible to identify the level of SPA and indicators of its structural components. To determine the level of empathy of students, we used the method of diagnosing empathic abilities of V.V. Boyko.

To establish changes in the level of indicators of the SPA, its structural components and empathy, the testing procedure was carried out in two stages - In the first month of study and at the end of the first semester. Descriptive statistics were used to process the study results.

\section{Results}

In the process of adaptation, with unresolved intrapersonal tensions, one of the primary substitutions is escapism; it is to this scale from the methodology of K. Rogers and R. Diamond that we paid attention to. Avoiding problem solving can be expressed in fantasizing non-existent events or engaging in activities that capture all the attention of an individual, creating comfortable conditions for him, distracting him from the perception of the course of real events. Z. Freud did not consider escapism to be a disease, but explained it as an additional element working to preserve the mental in a safe zone. However, being a protective mechanism and changing the perception of reality, escapism actualizes the regressive features of the behavior and response of a person to events taking place in reality. Therefore, the socialization of a person immersed in escapism can pass into the stage of a developmental crisis.

At the first stage, the study showed the following average values of SPA and its indicators among students: adaptation - average level (71 per cent); empathy - underestimated level 
(19 points); escapism - borderline value between low and medium level (10 points); variability of solutions to situational problems -3.5 points (on a five-point scale of marks).

The process of longitudinal observation of the productivity of online learning showed a decrease in the communicative activity of students in the classroom from the beginning of the academic year to the end of the first semester, inaccurate performance of tasks, little initiative in finding solutions to problems, waiting for solutions by other students or refusing to solve them.

Despite the active communication in social networks, the students did not demonstrate the dynamics of the development of the variability of solutions to situational problems in the learning process. The digital environment cannot replace the completeness of natural interpersonal communication.

The second stage of the study ascertained changes in students' indicators of the level of SPA and its indicators (empathy and escapism). The following average values were obtained: adaptation - average level (64 per cent); empathy - underestimated level (16 points); escapism - medium level (14 points); variability of solutions to situational problems - 3.0 points (on a five-point scale of marks). Obviously, there is a decrease in the level of SPA along with a decrease in the level of empathy and an increase in the level of escapism, which leads to a decrease in the variability of solving situational problems of all levels of complexity. Changes in indicators are shown in Figure 1.

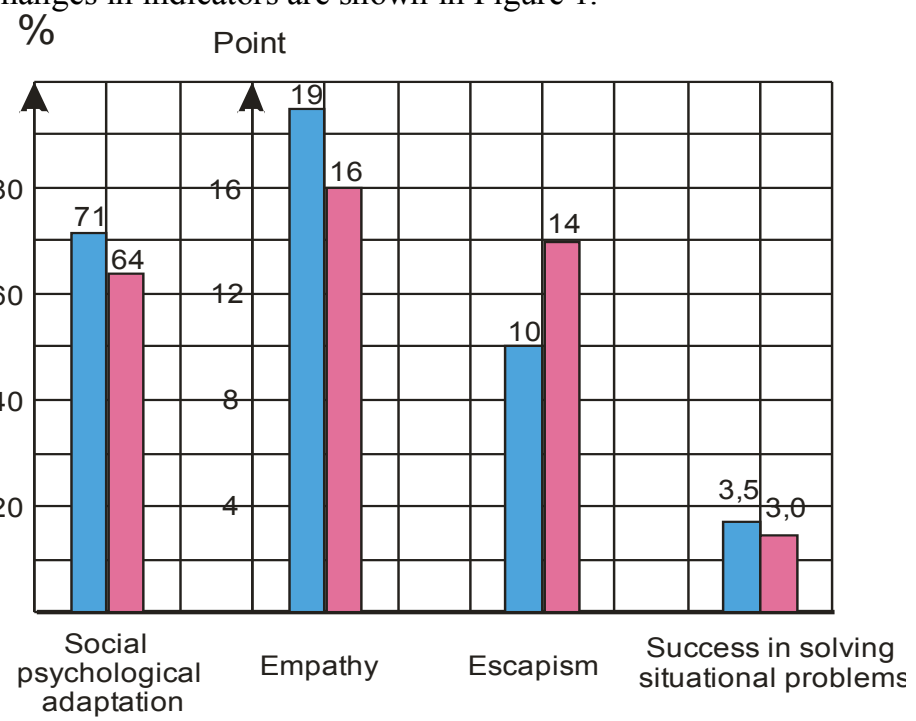

Fig. 1. Changes in indicators of social and psychological adaptation.

To establish the influence of social deprivation on the level of escapism and empathy using a statistical analysis of the research results, we proposed an integral indicator (A), which is calculated by the formula:

$$
\begin{gathered}
A=(E m p-E s c)_{1}-(E m p-E s c)_{2}=\left(E m p_{1}-E m p_{2}\right)-\left(E s c_{1}-E s c_{2}\right)= \\
=\Delta E m p-\Delta E s c
\end{gathered}
$$

Index 1 corresponds to the difference in the values of indicators of empathy and escapism before the conditions of deprivation, and index 2 - after the conditions of deprivation.

It was found that the integral indicator $A$ in all cases, without exception, demonstrates an increase in the difference in the values of empathy and escapism before and after the appearance of conditions of deprivation. 
At the same time, the variability of solving situational tasks at the middle level (one solution) almost did not change (from 56 to 64 per cent), and the "extreme" indicators (several solutions or no solution at all) worsened (36 per cent) (Table 1).

Table 1. Changing the degree of success of situational tasks.

\begin{tabular}{|c|c|c|c|}
\hline \multirow[b]{2}{*}{ № } & \multirow[b]{2}{*}{$\begin{array}{c}\text { Success rate of problem } \\
\text { solving }\end{array}$} & \multicolumn{2}{|c|}{ Number of students, (per cent) } \\
\hline & & $\begin{array}{c}\text { Before the appearance of } \\
\text { conditions of social } \\
\text { deprivation }\end{array}$ & $\begin{array}{l}\text { In conditions of } \\
\text { social deprivation }\end{array}$ \\
\hline 1 & $\begin{array}{l}\text { Showed several solutions to } \\
\text { a situational task }\end{array}$ & 38 & 18 \\
\hline 2 & $\begin{array}{l}\text { Showed one solution to a } \\
\text { situational task }\end{array}$ & 56 & 64 \\
\hline 3 & $\begin{array}{l}\text { Didn't solve the situational } \\
\text { task }\end{array}$ & 6 & 18 \\
\hline
\end{tabular}

\section{Discussion}

The results of the study confirmed our assumption about changes in students' levels of empathy, escapism and SPA in general. The change in the indicators of the structural components of SPA and empathy gives an idea of the decrease in the success of adaptation and the variability of solutions to psychological problems among students in the online learning process.

The experience of deprivation by a person, as a rule, is associated with the peculiarities of the social environment that surrounds person. Today, the most pressing problem in society is the problem of forced self-isolation of citizens in connection with the corona virus pandemic. Based on the opinion of a number of researchers (Yu.A. Marenchuk, O.I. Noskov, S.Yu. Rozhkov, and A.A. Avanesyan), it can be argued that life in extreme or stressful conditions leads to changes in cognitive, affective and behavioral spheres of individual and group consciousness [19].

Social deprivation often leads to negative mental conditions and emotions: fear, anxiety, depression. Similar conditions occur in people who are forced to be alone for a long time in extreme or stressful conditions, for example, travelers crossing the ocean alone, people in prison, at home in forced self-isolation, including in connection with restrictive measures in a pandemic situation etc. This situation brought with it a lot of sorrow and difficulties, but, firstly, as V.V. Kozlov noted, all the inhabitants of the planet have united "into one social body... for the first time in evolution, humanity acted as a subject as a mega-system in relation to the phenomenon of the COVID-19 pandemic" [3, p. 93], which cannot but portend the restoration of humanity; secondly, it forcedly promoted medical achievements; thirdly, it made it possible for scientists from various scientific fields to conduct research. These studies related to the analysis of politics and economics in global and individual values [20], the influence of the media on the degree of adherence to isolation, the virus distribution of information, people's understanding of the risks of infection, the formation of prejudices [21], [22], [23], the creation of hybrid machine learning models, SIRNet, to predict the spread of the COVID-19 pandemic in combination with existing epidemiological models, and others [24].

In this simultaneously being and research field, the most interesting are the psychological aspects of isolation, and hence of deprivation. It is obvious that today people are increasingly experiencing a sense of fear, constant anxiety, panic attacks, a desire to remain in isolation, a tendency to autism. In conditions of deprivation, a person's routine often changes, the level of dissatisfaction with the environment rises, many people lose control over themselves, as 
well as the meaning and purpose of life, as a result of such conditions, apathy and passivity arise. Scholars S.N. Yaremenko, L.O. Pazin, L.G. Babakhova, exploring these conditions, in her works reveal the features of apathetic human behavior in conditions of self-isolation: "apathetic behavior manifests itself in the form of a passive refusal of a modern person on the conditions of existence offered by the consumption system" [25, p. 16]. As opposed to (or a continuation) of apathetic behavior, we can talk about the growth of aggressiveness of a person who is in isolation.

This article presents only part of the results. The diagnostic technique of SPA by K. Rogers and R. Diamond makes it possible (which will be presented in other publications) to show changes at the statistical level also in terms of such indicators as adaptability disadaptivity, self-acceptance - self-rejection, acceptance of others - rejection of others, emotional comfort - emotional discomfort, internal control - external control, dominance submission.

\section{Conclusions}

The study confirmed the hypothesis put forward about possible changes in the level of empathy, the ability to solve situational tasks and SPA in general among students studying remotely. The level of empathy and SPA decreased (although the level of empathy remained underestimated, and the level of SPA was average, we see a tendency to a decrease in the results): at the first stage: adaptation - the average level (71 per cent); empathy underestimated level (19 points); escapism - borderline value between low and medium level (10 points); variability of solutions to situational problems -3.5 points (on a five-point scale of marks). At the second stage: adaptation - medium level (64 per cent); empathy underestimated level (16 points); escapism - medium level (14 points); variability of solutions to situational problems -3.0 points (on a five-point scale of marks). It is also obvious that the ability to solve situational problems decreases, the level of escapism from the borderline "low-average" goes to the average. The revealed trends allow us to assert that the conditions of self-isolation and distance learning - and there is deprivation in all its aspects - Is not only social, but also sensory, emotional, cognitive and motor. The problem undoubtedly requires the attention of researchers, and no less of practical psychologists and psychotherapists. The main point - not only students with a plastic psyche, but also teachers with all their significant experience, faced very complex problems of deprivation.

\section{References}

1. J. Langmeyer, Z. Matejchek, Mental deprivation in childhood (Avicenum, Medical Publishing House Prague, 1984).

2. E.G. Alekseenkova, Personality in conditions of mental deprivation (Peter St. Petersburg, 2009).

3. V.V. Kozlov, Human factor: social psychologist. 2 (40), 93-103 (2020).

4. F.B. Berezin, Mental and psychophysiological adaptation of a person (Nauka Publishing House, Leningrad, 1988).

5. T.P. Vetlugina, V.B. Nikitina, T.I. Nevidimova, Basic research 9 (1), 17-21 (2012).

6. T. Shibutani, Society and personality: An interactionist approach to social psychology (Prentice-Hall, Inc., 1961).

7. A. Freud, The Ego and the Mechanisms of Defence (Karnac Books Ltd., 2018).

8. S. Freud, The Ego and the Id. Create Space (Independent Publishing Platform, 2010). 
9. C.G. Jung, Modern Man in Search of a Soul (Harvest: pbk., 2017).

10. H. Hartmann, Intl Universities Pr Inc. (1958).

11. A.A. Nalchadjyan, Psychological adaptation: mechanisms and strategies (Eksmo Publishing House, Moscow, 2010).

12. G. Selye, Stress without distress (Vieda Publishing House Riga, 1992).

13. A.A. Rean, Psychology of personality. Socialization, behavior, communication (St. Petersburg: Prime-Evroznak Publishing House; Moscow: Olma-Press Publishing House, 2004).

14. K.R. Rogers, A theory of therapy, personality and interpersonal relationships as developed in the clientcentered framework, Psychology: A study of a science. Vol. III: Formulations of the Person and the Social Contects, New York, McGraw-Hill Book Company, 1959).

15. W. Fei, Yi. Geng, Sh. Wang, Qi. Ma, X. Peng, M. Zhang, T. Zhang, Journal of Affective Disorders. Available online 8 March 2021 In Press. Journal Pre-proof What are Journal Pre-proof articles? URL:https://www.sciencedirect.com/science/article/abs/pii/S0165032721002305?dgci $\mathrm{d}=\mathrm{rss} \_$sd_all.

16. M.C.M. Wever, L.A.E.M. Van Houtum, L.H.C. Janssen, G-J. Will, M.S. Tollenaar, M.E. Bernet B.M. lzinga, Neuro Image 232, 117886 (2021).

17. J. Zaki, On the good side. The power of empathy in a fragmented world (Publishing House "Mann, Ivanov and Ferber" Moscow, 2020).

18. D.Ya. Raigorodsky, Practical psychodiagnostics. Techniques and tests (Publishing House "BAHRAKH" Samara, 2011).

19. Yu.A. Marenchuk, O.I. Noskov, S.Yu. Rozhkov, A.A. Avanesyan, Psychological aspects of personality in emergency situations. Psychological safety of the individual in extreme conditions and crisis situations of life: $\mathrm{V}$ international scientific and practical conference (Vladivostok, 87-91, 2015).

20. C.M. Giancarlo, L.G. Bodenstein, Center for Economic Policy Research. 04.17.2020.

21. P. Block, M. Hoffman, I.J. Raabe, J.B. Dowd, Ch. Rahal, R. Kashyap, M.L. Mills, Physics and Society. 15 April 2020. URL: https://arxiv.org/abs/2004.07052.

22. D.S. Jones, M.D. History in a Crisis - Lessons for Covid-19. April 30, 2020. N Engl J Med; 382: 1681-1683 (2020). DOI: 10.1056 / NEJMp2004361. URL: https://www.nejm.org/doi/full/10.1056/NEJMp2004361?query=recirc_artType_railA_ article\#article_references.

23. I. Zagury-Orly, B. Sc, R.M. Schwartzstein, M.D. Covid-19 - A Reminder to Reason, (2020). DOI: $\quad 10.1056 \quad$ NEJMp2009405. URL:https://www.nejm.org/doi/full/10.1056/NEJMp2009405.

24. N. Soures, D. David Chambers, Z. Carmichael, A. Daram, D.P. Shah, K. Clark, L. Potter, D. Dhireesha Kudithipudi, Populations and Evolution. 22 Apr 2020. URL: https://arxiv.org/abs/2004.10376v1.

25. L.O. Yaremenko, L.O. Pazina, L.G. Babakhova, Sociocultural habit of apathy in a transitive society (monograph, Rostov-on-Don, 2010). 